\title{
Les perspectives de thérapie génique de la mucoviscidose
}

Le gène de la mucoviscidose code pour une protéine appelée CFTR (cystic fibrosis transmembrane conductance regulator) qui est fonctionnellement liée aux mouvements de chlore au travers de la membrane plasmique. Les cellules de l'épithélium bronchique et du pancréas des malades ne répondent pas comme elles le devraient à la stimulation par l'AMP cyclique en augmentant le flux sortant de $\mathrm{Cl}^{-}$ (anion chlore). Ce mouvement ionique est normalement accompagné d'eau, si bien que les sécrétions bronchiques et pancréatiques des sujets atteints sont anormalement épaisses, entraînant les complications connues d'obstruction et d'ectasie bronchiques se compliquant de surinfections qui font toute la gravité de la maladie, ainsi que, inconstamment, d'insuffisance pancréatique exocrine. Une première étape à franchir avant que d'envisager une approche de thérapie génique était de montrer que l'ADNc de la protéine CFTR, intégré dans un vecteur d'expression, commandait bien la synthèse d'une protéine fonctionnelle capable de corriger ex vivo (en culture de cellules) le désordre du mouvement de chlore des cellules de malade. L'ADNc complet de CFTR fut en fait difficile à cloner dans des bactéries car sa séquence comporte un motif reconnu comme un promoteur procaryotique dont l'activation est toxique pour les bactéries transformées, probablement parce qu'elle entraîne l'accumulation d'une protéine délétère pour le microorganisme. Deux équipes ont cependant réussi à obtenir ce clone, l'une en mutant la séquence promotrice [1] et l'autre en utilisant un vecteur à très faible nombre de copies dans la cellule, limitant ainsi l'abondance du produit toxique [2]. Exprimée dans des fibroblastes, CFTR apparaît être une protéine membranaire, glycosylée et phosphorylée, dont les liens avec le canal à chlore restent très incertains. Il pourrait s'agir d'une protéine contrôlant directement ou indirectement ce type de canal. La preuve définitive que les mutations de CFTR sont bien responsables de l'anomalie fonctionnelle a été apportée en infectant des cellules de malade à l'aide de rétrovirus commandant l'expression de l'ADNc et en démontrant que l'expression de CFTR corrigeait l'anomalie des mouvements de chlore après stimulation par l'AMPc [1, 3].

Quelle route reste-t-il à parcourir pour aller de cette première étape aux premiers essais de thérapie génique chez l'homme ? Il est probable que la correction du déficit au niveau du poumon serait de nature à transformer le pronostic de la maladie, comme le montrent les très bons résultats de la greffe pulmonaire lorsqu'elle n'est pas rejetée. On ne connaît cependant pas, à ce jour, les cellules dont la correction serait indispensable à l'obtention d'un résultat. De toute façon, il n'est pas question ici d'utiliser une autogreffe de cellules génétiquement modifiées par culture ex vivo, comme cela est envisagé pour la moelle osseuse [4] et pour d'autres types cellulaires [5]. La seule possibilité semble être le transfert in vivo. Pour ce faire, trois types de vecteurs peuvent être envisagés. Tout d'abord, les liposomes, qui sont des vésicules lipidiques artificielles pouvant englober du matériel divers, dont de l'ADN. Les résultats actuels du transfert in vivo et ex vivo d'ADN par ce moyen ne sont pas tous convaincants, mais on peut penser qu'une administration par nébulisation intra-trachéo-bronchique constituerait de bonnes conditions pour ce type de transfert. Les vecteurs rétroviraux viennent ensuite à l'esprit, mais nous avons déjà dit les interrogations qui subsistaient quant à leur utilisation par application directe in vivo [5] ; de plus, les rétrovirus n'infectent que les cellules en cours de division, ce qui n'est pas forcément le cas des cellules bronchiques qu'il faudrait corriger. L'emploi d'adénovirus recombiné, enfin, semble assez attractif. Cet agent a démontré sa potentielle efficacité pour corriger, après administration in vivo, une maladie héréditaire, le déficit en ornithine transcarbamylase $[6,7]$. Il est normalement un agent infectieux, le plus souvent bénin, des voies aériennes et peut parfaitement infecter des cellules qui ne se divisent pas. Un premier essai d'infection in vivo de la trachée de rat par un adénovirus de type recombiné a enfin démontré que les cellules infectées sécrétaient la protéine codée par le gène intégré dans le génome viral [8]. Un autre virus utilisable serait l'AAV (adeno-associated virus) virus défectueux (defective) ne pouvant croître sans auxiliaire et dépourvu de pathogénicité $[9,10]$. Les obstacles restent cependant considérables avant que l'on puisse vraiment traiter les malades atteints de mucoviscidose par transfert de gène. L'un est l'absence de modèle animal, pourtant indispensable à la mise au point de ce type de traitement. La solution à cette difficulté serait probablement l'inactivation du gène endogène de souris par recombinaison homologue [11]. Il semble cependant que cette recombinaison homologue au niveau du gène CFTR se soit révé- 
lée très difficile pour l'instant. Quand un tel résultat sera obtenu, restera à déterminer si la symptomatologie des souris homozygotes pour la mutation mimera la maladie humaine. Un autre problème potentiel est la probable nécessité dans laquelle on se trouvera de répéter les transferts géniques par pulvérisation d'aérosols. Il est en effet improbable que soient infectées toutes les cellules souches à l'origine des cellules cibles du transfert de gène, si bien que les cellules infectées éliminées par desquamation bronchique et vieillissement cellulaire pourraient être remplacées par des cellules ne possédant par le génome viral. On peut donc se demander si la répétition d'apports de particules virales ne créera pas une immunisation dont les conséquences pourraient être une baisse de l'activité thérapeutique, voire des phénomènes immuno-allergiques broncho-pulmonaires aggravant la fonction respiratoire des malades.
Enfin persiste toujours la possibilité théorique d'une recombinaison entre le virus défectueux utilisé comme vecteur et un virus "sauvage " normal aboutissant à la libération de particules virales infectieuses transportant le gène CFTR intégré partout dans l'organisme.

Ainsi peut-on dire que les récents développements dans la génétique moléculaire de la mucoviscidose ont élevé la thérapie génique au rang de traitement possible de la maladie... possible mais pas certain, possible mais lointain.

1. Drumm ML, Pope HA, Cliff WK, et al. Correction of the cystic fibrosis defect in vitro by retrovirus-mediated gene transfer. Cell $1990 ; 62: 1227-33$.

2. Gregory RJ, Cheng SH, Rich DP, et al. Expression and characterization of the cystic fibrosis transmembrane conductance regulator. Nature $1990 ; 347$ : 382-6.
3. Rich DP, Anderson MP, Gregory RJ, et al. Expression of cystic fibrosis transmembrane conductance regulator corrects defective chloride channel regulation in cystic fibrosis airway epithelial cells. Nature $1990 ; 347$ : 358-63. 4. Lehn P. Vecteurs rétroviraux pour le transfert de gènes dans le tissu hématopoiétique in vivo. médecine/sciences $1990 ; 6: 791-9$.

5. Kahn A, Briand P. Nouvelles orientations pour la thérapie génique. médecine/sciences 1990 ; 6 : 144-9.

6. Chasse JF, Levrero M, Kamoun P, et al. L'adénovirus: vecteur de thérapie génique? médecine/sciences $1989 ; 5: 331-7$.

7. Stratford-Perricaudet LD, Levrero $M$, Chasse JF, Perricaudet M, Briand P. Evaluation of the transfert and expression in mice of an enzyme-encoding gene using a human adenovirus vector. Hum Gen Ther $1990 ; 1$ : 241-56.

A.K. 8, Crystal RG, Rosenfeld M, Siegfried W, et al. New viral transfert systems. Pediatr Pulmo nol $1990 ; 5$ (suppl) : 103.

9. Carter BJ, Flotte TR, Owens RA. Adenoassociated viruses for gene transfer. Pediatr Pulmonol 1990 ; 5 (suppl) : 104-5.

10. Editorial : Cystic fibrosis : towards the ultimate therapy, slowly. Lancet 1990 ; 336 1224-5.

11. Lemarchandel V, Montagutelli X. La recombinaison homologue : de nouvelles perspectives pour la transgenèse. médecine/sciences $1990 ; 6: 18-29$ 